\title{
The Semantics of Power and ARM Multiprocessor Machine Code
}

\author{
Jade Alglave $^{2} \quad$ Anthony Fox ${ }^{1} \quad$ Samin Ishtiaq $^{3} \quad$ Magnus O. Myreen ${ }^{1}$ \\ Susmit Sarkar ${ }^{1} \quad$ Peter Sewell $^{1} \quad$ Francesco Zappa Nardelli ${ }^{2}$ \\ ${ }^{1}$ University of Cambridge $\quad{ }^{2}$ INRIA $\quad{ }^{3}$ Microsoft Research Cambridge \\ http://www.cl.cam.ac.uk/ pes20/weakmemory/
}

\begin{abstract}
We develop a rigorous semantics for Power and ARM multiprocessor programs, including their relaxed memory model and the behaviour of reasonable fragments of their instruction sets. The semantics is mechanised in the HOL proof assistant.

This should provide a good basis for informal reasoning and formal verification of low-level code for these weakly consistent architectures, and, together with our x86 semantics, for the design and compilation of high-level concurrent languages.
\end{abstract}

Categories and Subject Descriptors C.1.2 [Multiple Data Stream Architectures (Multiprocessors)]: Parallel processors; D.1.3 [Concurrent Programming]: Parallel programming; F.3.1 [Specifying and Verifying and Reasoning about Programs]

General Terms Documentation, Reliability, Standardization, Theory, Verification

Keywords Relaxed Memory Models, Semantics, PowerPC, ARM

\section{Introduction}

Parallelism is finally going mainstream, but, despite 40 years of research on concurrency, programming and reasoning about concurrent systems remains very challenging. A key issue is that most research has implicitly assumed that memory is sequentially consistent, whereas the reality is that typical multiprocessor architectures -including x86, Sparc, Power, Itanium, ARM, and Alpha - only provide relaxed (or weak) memory models. For performance reasons, their implementations involve out-of-order execution (reordering operations within processor pipelines), and multiple levels of caching and write buffering, to reduce latency. These microarchitectural optimizations have observable effects at the assembly language level: different processors can see the events of a complete execution in different orders; they can

Permission to make digital or hard copies of all or part of this work for personal or classroom use is granted without fee provided that copies are not made or distributed for profit or commercial advantage and that copies bear this notice and the full citation on the first page. To copy otherwise, to republish, to post on servers or to redistribute to lists, requires prior specific permission and/or a fee.

DAMP'09, January 20, 2009, Savannah, Georgia, USA.

Copyright (C) 2009 ACM 978-1-60558-419-5/09/01 . . \$5.00 have inconsistent views of the shared memory. It would not be sound to think about such a system in terms of an intuitive interleaving semantics, in which the events from different processors act on a single global state. Instead, we need to understand the subtle guarantees that each architecture provides, both for low-level programming, including implementation of concurrency libraries and OS or hypervisor kernels, and for designing the new high-level concurrent languages that are so urgently needed, so that they can be efficiently compiled down to these architectures.

Previous work on multiprocessor memory models has addressed various more-or-less idealised architectures, and several tutorials have been published [AG96, Luc01, LJV97]. However, typical vendor specifications are still expressed only in informal prose, supplemented by a few litmus-test examples of small programs. Inevitably this is ambiguous and incomplete. The Itanium and SPARC have vendor specifications in semi-formal mathematics [Ita, Spa], but these still leave ample room for interpretation [HJK06, YGLS04, PD95], and the situation for $\mathrm{x} 86$, Power, and ARM leaves a great deal of scope for confusion. Moreover, none of the previous work gives a complete semantics for multiprocessor programs, as it does not integrate the memory models with semantics for instructions.

In this paper we describe a semantics for multiprocessor Power and ARM programs, in a declarative axiomatic style. It is precise, formalised in the HOL proof assistant [HOL]. The memory model (in Section 2) is integrated with instruction semantics and decoding (in Section 3) for reasonable fragments of the instruction sets, including various ALU operations, branches, loads and stores, reservations, and barriers (we do not model page tables, access to device memory, or exceptions). We also discuss a number of litmus tests (Section 4) and some initial empirical testing against particular processors (Section 5). We believe the semantics to be reasonably accurate, but this is work in progress: in future work we plan more extensive testing, and we welcome feedback from programmers and architects with experience in these architectures. We plan also to prove metatheoretic results, as in our complementary work on x86-CC $\left[\mathrm{SSZN}^{+} 09\right]$, where we proved results about data-race-free programs and an abstract-machine version of the memory model.

Such semantics should provide a good basis for informal reasoning about the majority of low-level concurrent algorithms, for formal verification and model-checking of such algorithms, and for the design of high-level language support for concurrency, all above the real multiprocessor architectures that we have today. 


\section{The Power/ARM Axiomatic Memory Model}

Our memory model is in a axiomatic style, specifying the legal orders of events in a valid execution of a multiprocessor program, as in previous formal work on relaxed memory models. A single instruction may involve a non-atomic collection of several reads or writes, so one cannot simply reorder whole instructions. Instead one has to work at the finer granularity of events that are individual reads and writes to memory. Our events also include individual reads and writes to processor registers (in contrast to most previous work), as the interplay between dependencies through registers and through memory is a key aspect of the model, and as the instruction semantics must involve register accesses.

We aim to capture Power and ARM architectures, rather than the behaviour of particular devices. The architectures are specifications of what can be relied upon by assemblylevel programmers, for whole families of past and (generally) future processors. They change relatively slowly, and are very loose specifications, to admit a wide variety of processor implementations. Our Power definition is based primarily on the Power ISA Version 2.05 specification [Pow07] (applicable to POWER6 and POWER5 processors), particularly Book II Ch.1, §3.4, and Appendix B, together with articles [SF95, LHF05], and reference to the PowerPC Book E [IBM02]. Our ARM definition is based upon the ARM Architecture Reference Manual [ARM08a] (applicable to ARMv7 processors), particularly $\S A 3.8$, and the Barrier Litmus Test Cookbook [ARM08b].

We choose to address Power and ARM for several reasons. First, they are both widely used: POWER processors in high-end servers, and both PowerPC and ARM in embedded devices. Even the latter are expected to become multicore, for power/performance reasons, e.g. with the ARM11 MPCore (up to 4 cores) and ARM Cortex-A9 MPCore.

Second, they give an interesting contrast to our $\mathrm{x} 86$ work. The current informal-prose $\mathrm{x} 86$ vendor specifications, formalised in our x86-CC memory model $\left[\mathrm{SSZN}^{+} 09\right]$, are, very roughly, causal consistency: $\mathrm{x} 86-\mathrm{CC}$ has a single transitively closed happens-before relation, which has to be respected in all processors' views, and very strong "LOCK"'d instructions, which are atomic and are seen in the same order by all processors. ${ }^{1}$ The Power memory model, on the other hand, is weakly consistent. It allows more local reordering, and provides load-reserve/store-conditional and a variety of barrier primitives. The ARM memory model is very close to that for Power, differing only in the barrier semantics.

Third, there is little previous literature on memory models for these architectures. For PowerPC there are models by Corella et al. [CSB] and Adir et al. [AAS03]; for ARM there is the initial work of Chong and Ishtiaq [CI08].

Fourth, there has been extensive work on precise semantics for both PowerPC and ARM in the single-processor case. Leroy's verified compiler research [Ler06] is founded on a Coq model of PowerPC instructions, and Fox has verified correspondence between an earlier ARM ISA specification and a microarchitectural model, in HOL [Fox03].

For both architectures, we aim to cover the fragment required for typical low-level concurrent algorithms in main memory, as they might appear in user or OS kernel programs. We do not deal with explicit manipulation of page

\footnotetext{
${ }^{1}$ It appears that actual x86 processors provide an even stronger TSO-based memory model, and the vendor specifications are expected to change to reflect that $\left[\mathrm{SSZN}^{+}\right.$09, Addendum].
}

tables, cache hints, self-modifying code, and so forth, and for Power at present we cover only one of the barrier instructions (sync 0, but not lwsync, eieio, mbar, or isync).

Our definitions are mechanised in the HOL proof assistant $[\mathrm{HOL}]$, and the axiomatic memory model definitions are also mechanised in the Coq proof assistant [Coq], handtranslated from the HOL. For lack of space only key extracts are included here, but the full details are freely available on-line [wea08]. Most of the definitions in this paper are automatically typeset from the HOL, reducing the scope for error.

\section{$2.1 \quad$ Basic Types}

The basic types of our model, introduced below, describe what a candidate execution is. They are very similar to those we used for $\mathrm{x} 86$, which makes it easier to compare the three models. It also lets us reuse tools, as we discuss in $\S 5$.

We take types address and value to both be the 32-bit words, and take a location to be either a memory address or a register of a particular processor. The memory model is polymorphic on a type 'reg of registers, later instantiated to the Power or ARM registers as appropriate. There are also pseudo-locations LOCATION_RES and LOCATION_RES_ADDR used to express the semantics of reservations $(\S 2.7)$.

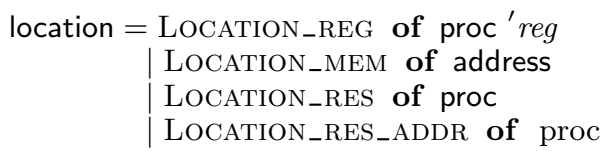

These constructors are curried, so LOCATION_REG : proc $\rightarrow$ ${ }^{\prime} r e g \rightarrow$ location. To identify an instance of an instruction in an execution, we specify its processor and its index in program order (i.e., in the program with an unfolding of all branches):

iiid $=\langle$ proc : proc;
poi : num $\rrbracket$

An action is either a read or write of a value at some location, or a synchronisation barrier (used as a marker to define the Power sync and the ARM DMB):

$$
\begin{aligned}
& \text { dirn }=\mathrm{R} \mid \mathrm{W} \\
& \text { synchronization }=\text { SYNC } \\
& \text { action = ACCESS of dirn ('reg location) value } \\
& \text { | BARRIER of synchronization }
\end{aligned}
$$

A typical action might be ACCESS W (LOCATION_MEM 100) 5, for a write of 5 to memory address 100 . Finally, an event has an instruction instance id, an event id (of type eiid = num, unique among the events of this iiid), and an action:

$$
\begin{aligned}
\text { event }= & \llbracket \text { eiid }: \text { eiid; } \\
& \text { iiid }: \text { iiid; } \\
& \text { action }: \text { action } \rrbracket
\end{aligned}
$$

The semantics of a single instruction must also record any intra-instruction causality relationships among its events. The Power and ARM architectures make a subtle distinction between causality due to data dependency, e.g. a write to an address (or of a value) that was taken from a register, and a control dependency, e.g. a write that was conditional on a particular flag value.

For example, the Power instruction lwz GPR2,0,GPR1 below, loading register GPR2 with the value from memory at the location in register GPR1, has an event structure 
with a register read, a memory read, and a register write, all linked by intra-instruction-causality-data:

\begin{tabular}{|c|c|}
\hline ppc-lwz & proc:0 \\
\hline poi:0 & lwz GPR2,0,GPR1 \\
\hline Initial st & te: $0: G P R 1=100$ \\
\hline
\end{tabular}

\begin{tabular}{|c|c|}
\hline \multicolumn{2}{|c|}{$\begin{array}{l}\text { eiid:0 (of lwz GPR2,0,GPR1) } \\
\text { iiid: }\langle\text { proc:0;po:0〉 } \\
\text { R 0:GPR1=100 }\end{array}$} \\
\hline$\nabla$ & iico_data \\
\hline $\begin{array}{l}\text { eiid:1 (of lwz GPR2 } \\
\text { iiid: }\langle\text { proc:0;po: } 0\rangle \\
\text { R }[100]=5\end{array}$ & 2,0,GPR1) \\
\hline$\nabla$ & iico_data \\
\hline $\begin{array}{l}\text { eiid:2 (of lwz GPR2 } \\
\text { iiid: }\langle\text { proc:0;po:0 } 0 \\
\text { W 0:GPR2 }=5\end{array}$ & 2,0,GPR1) \\
\hline
\end{tabular}

ppc-lwz: (event structure 1)

The ARM LDRNE R1, [R10], on the other hand, conditionally loads register $\mathrm{R} 1$ with the value of memory at the location in R10, if flag EQ is zero.

\begin{tabular}{|l|c|}
\hline ARM-ldrne & proc:0 \\
\hline poi:0 & LDRNE R1, [R10] \\
\hline
\end{tabular}

It therefore has two families of event structures - those in which there is a single event, reading a non-zero flag value, and nothing else happens:

$$
\begin{aligned}
& \text { eiid:0 (of LDRNE R1, [R10]) } \\
& \text { iiid: }\langle\text { proc:0;po:0 } \\
& \text { R 0:EQ=1 } \\
& \multicolumn{1}{|c|}{\text { ARM-ldrne: (event structure 2) }}
\end{aligned}
$$

and those in which it reads a zero flag value, with an intrainstruction-causality-control relation to the read of R10:

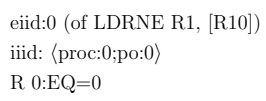

Collecting this data together, we define an event structure $E$ to comprise a set of events, an intra-instruction data causality relation, and an intra-instruction control causality relation. It also specifies an architecture (POWER205 or ARMv7, see $\S 2.5$ ), and a reservation granule size and atomicity relation (see $\S 2.7$ ).

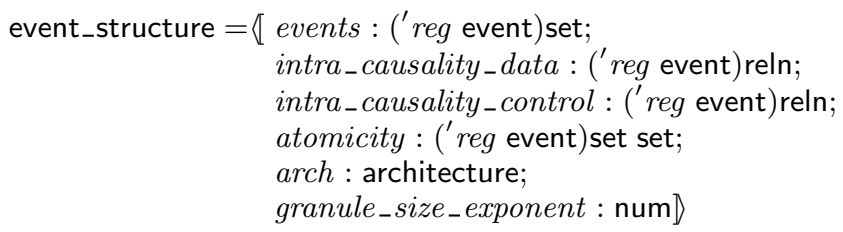

This is subject to various well-formedness conditions (such as that the event pairs in the intra-causality relation are, indeed, elements of the event set), which we omit here.

Given an event structure, a candidate execution witness consists of an initial state constraint and a processor-indexed family of view orders, together with a write serialization that we explain in $\S 2.3$. The rest of this section is devoted to defining when such a candidate is in fact a valid execution.

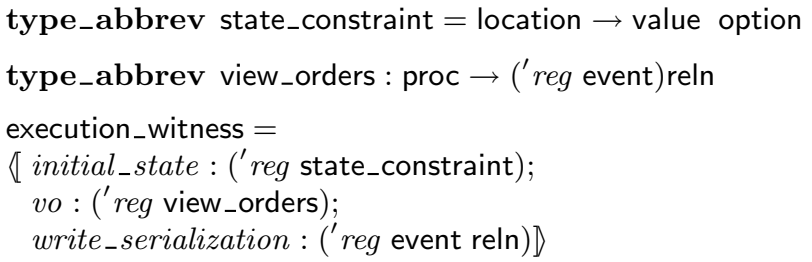

A well-formed view order for processor $p$ is a strict linear order over all of its events together with all the memory write events of other processors; we write viewed_events $E p$ for that union.

\subsection{Read Values}

Our first condition simply says that any read event, of a memory or register location (or a reservation address), reads the value either of the most recent write to that location, if there is one, or otherwise from the initial state. Here "most recent" is with respect to the view order of the reading processor. The state_updates auxiliary finds the write events before a given event $e$ that write to the same location.

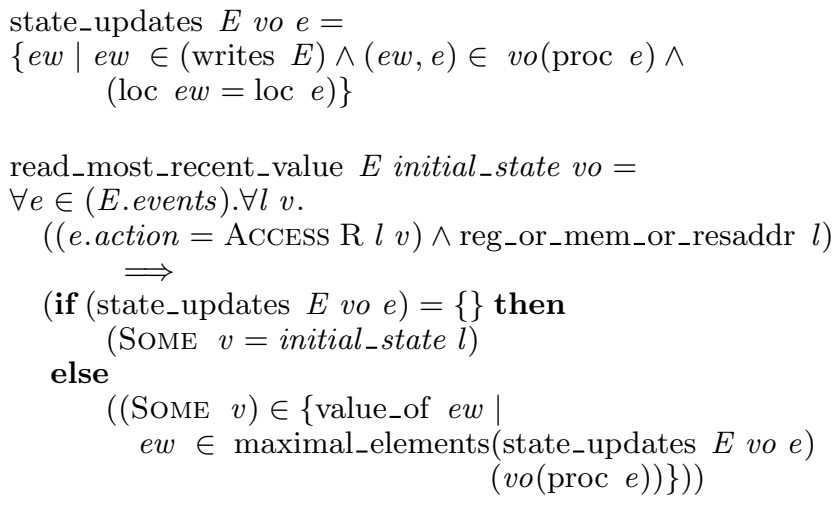

\subsection{Coherence}

Both architectures (when in the appropriate mode) provide coherent memory: writes by two processors to the same memory location must be seen by all processors in the same order. In other words, for each memory location, there exists a strict linear order on the stores to this location, and this must be respected by all the view orders. We formalise this by defining the candidate write serializations, each of which 
is a union of a candidate order over the writes for each memory location.

get_mem_l_stores $E l=$

$\{e \mid e \in$ E.events $\wedge$ mem_store $e \wedge(\operatorname{loc} e=\operatorname{SomE} l)\}$

write_serialization_candidates $E$ cand $=$

$\left(\forall\left(e_{1}, e_{2}\right) \in\right.$ cand.

$\exists l . e_{1} \in($ get_mem_l_stores $E l) \wedge$ $e_{2} \in($ get_mem_l_stores $\left.E l)\right) \wedge$

$\left(\forall l\right.$.strict_linear_order $\left(\right.$ cand $\left.\left.\right|_{(\text {get_mem_l_stores } E l)}\right)$ (get_mem_l_stores $E l$ ))

We require that the write serialization of an execution is included in each processor's view order, and that the orderings of writes by each processor to the same location, captured with preserved_coherence_order below, are included in the write serialization.

preserved_coherence_order $E p=$

$\left\{\left(e_{1}, e_{2}\right) \mid\left(e_{1}, e_{2}\right) \in\right.$ po_iico_data $E \wedge$

$\left(\operatorname{proc} e_{1}=p\right) \wedge\left(\operatorname{proc} e_{2}=p\right) \wedge\left(\operatorname{loc} e_{1}=\operatorname{loc} e_{2}\right) \wedge$

(mem_store $e_{1} \wedge$ mem_store $\left.\left.e_{2}\right)\right\}$

Here po_iico_data is the union of the strict program order relation and the intra-causality-data relation:

po_strict $E=$

$\left\{\left(e_{1}, e_{2}\right) \mid\left(e_{1}\right.\right.$. iiid.proc $=e_{2}$.iiid.proc $) \wedge$

$e_{1}$.iiid.poi $<e_{2}$.iiid.poi $\wedge$

$e_{1} \in$ E.events $\wedge e_{2} \in$ E.events $\}$

po_iico_data $E=$ po_strict $E \cup E_{\text {.intra_causality_data }}$

po_iico_both $E=$ po_strict $E \cup$ E.intra_causality_data $\cup$ E.intra_causality_control

Note that coherence does not require that writes to two different locations must be seen by all processors in the same order.

\subsection{Preserved Program Order}

The essence of a weakly consistent model, such as those of Power or ARM, is that a processor implementation is free to re-order its own operations rather liberally, in between synchronisation operations, so long as dependencies are respected - any reordering not forbidden is permitted. The dependencies that are respected, however, are subtly less than what one might expect, and we need to take several steps to define them. Moreover, the documentation speaks of dependencies between instructions, but again we actually need to deal in terms of dependencies between events, using the intra-instruction causality as appropriate.

First, we identify the data dependencies through the registers of a single processor, picking out the pairs of events in program order (strictly, in po_iico_data), on a processor $p$, where $e_{1}$ is a write to a register and $e_{2}$ is a read from the same register, with no intervening write to the same register:

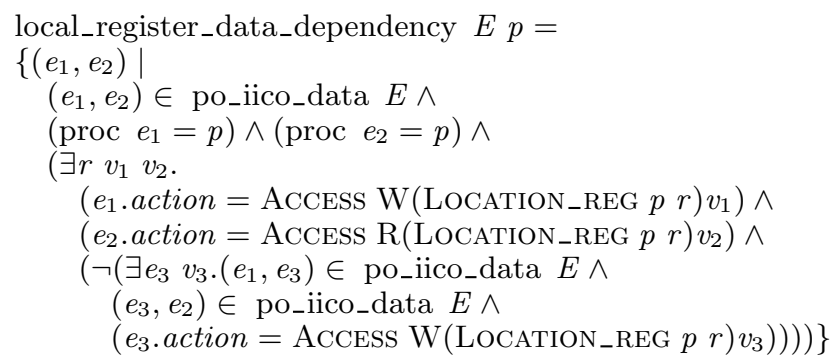

For example, in the short ARM program below, there is a local register data dependency from the write of $\mathrm{R} 1$ in the first instruction to the read of $\mathrm{R} 1$ in the second.

\begin{tabular}{|l|c|}
\hline ARM-depend-A & proc:0 \\
\hline poi:0 & LDR R1, [R0] \\
poi:1 & LDR R2, [R1] \\
\hline
\end{tabular}

A sample event structure for that program is shown below. Note that the intra-instruction causality relations are part of the event structure data, provided by the instruction semantics, whereas local register data dependency (lrdd) is calculated from the event structure and candidate execution.

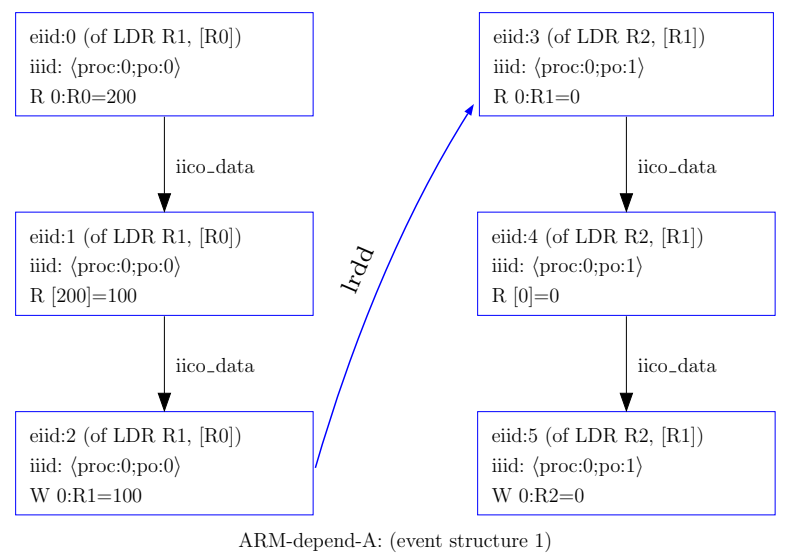

For dependencies from a memory load to a memory load, we pick out the pairs $\left(e_{1}, e_{2}\right)$ of two memory loads, in program order and by the same processor (though perhaps to different addresses), which are transitively related by the union of local register data dependency and the intra-instruction data causality relation (perhaps counterintuitively, the intra-instruction control relation is not included):

$$
\begin{aligned}
& \text { address_or_data_dependency_load_load } E p= \\
& \left\{\left(e_{1}, e_{2}\right) \mid\right. \\
& \quad\left(\text { mem_load } e_{1} \wedge \text { mem_load } e_{2} \wedge\left(e_{1}, e_{2}\right) \in \text { po } E \wedge\right. \\
& \quad\left(\text { proc } e_{1}=p\right) \wedge\left(\text { proc } e_{2}=p\right) \wedge \\
& \quad\left(e_{1}, e_{2}\right) \in((E \text {.intra_causality_data } \cup \\
& \left.\left.\left.\quad \text { local_register_data_dependency } E p)^{+}\right)\right)\right\}
\end{aligned}
$$

Here $r^{+}$denotes the transitive closure of a relation $r$.

For example, in the ARM program below, the LDRNE instruction is conditional on the EQ flag.

\begin{tabular}{|l|l|}
\hline ARM-nodep & \multicolumn{1}{|c|}{ proc:0 } \\
\hline poi:0 & LDR R1, [R4] \\
poi:1 & CMP R1, \#1 \\
poi:2 & LDRNE R2, [R5] \\
\hline
\end{tabular}

Even if the value read from that flag is 0, as in Fig. 1, there is no address_or_data_dependency_load_load from the first memory load to the memory load of the LDRNE.

However, in the program below [ARM08b, $§ 6.2 .1 .2 \mathrm{~b}$ ] there is an address_or_data_dependency_load_load from the first memory load to the second memory load, despite the fact that this is a "false" dependency, i.e. the address used by the second load is not affected by the value read 


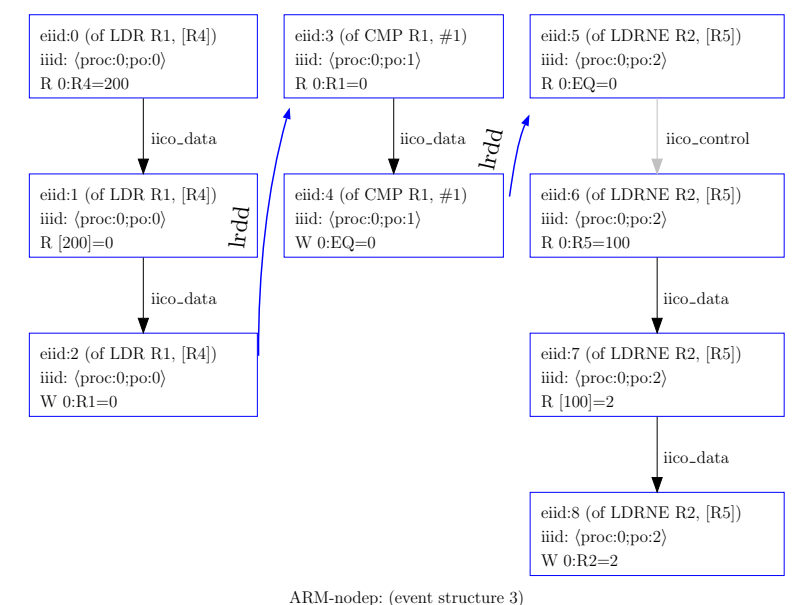

Figure 1.

by the first. Introducing such "false" dependencies can be useful to constrain execution orders.

\begin{tabular}{|l|l|}
\hline ARM-depend-B & proc:0 \\
\hline poi:0 & LDR R1, [R0] \\
poi: 1 & AND R1, R1, \#0 \\
poi:2 & LDR R2, [R3,R1] \\
\hline
\end{tabular}

Dependencies from a memory load to a memory store are similar, except that here both intra-instruction relations are included:

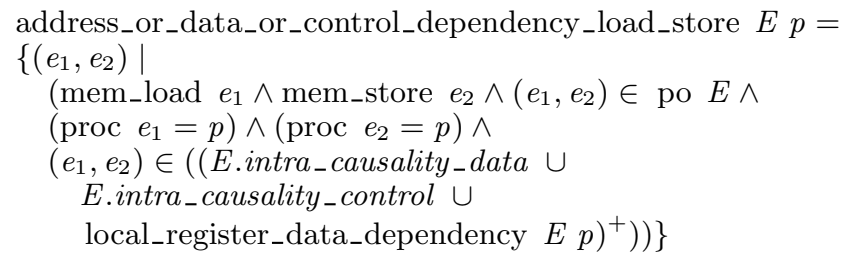

The program below [ARM08b, §6.2.1.2c, modified with a STR instead of a LDR at the end] illustrates such an address_or_data_or_control_dependency_load_store, passing through the intra-instruction-control relation. In this case, the execution (or not) of the memory store depends on the value returned by the memory load.

\begin{tabular}{|l|l|}
\hline ARM-depend-C & \multicolumn{1}{|c|}{ proc:0 } \\
\hline poi:0 & LDR R1, [R0] \\
poi:1 & CMP R1, \#55 \\
poi:2 & STRNE R2, [R3] \\
\hline
\end{tabular}

The next example [ARM08b, §6.2.1.2d, likewise modified] is similar, except that here the result of the conditional MOV influences the address to which the store access is performed.

\begin{tabular}{|l|l|}
\hline ARM-depend-D & \multicolumn{1}{|c|}{ proc:0 } \\
\hline poi:0 & LDR R1, [R0] \\
poi:1 & CMP R1, \#55 \\
poi:2 & MOVNE R4, \#0 \\
poi:3 & STR R2, [R3,R4] \\
\hline
\end{tabular}

Note that the semantics does not require the concept of a pure control dependency.
We now turn to the special case of memory accesses to the same address. Here we include all such pairs: omitting load/load or load/store would make a nonsense of the coherence property; store/store is also enforced by coherence, so harmless to include here; and without store/load a load could fail to read from a program-order-past store:

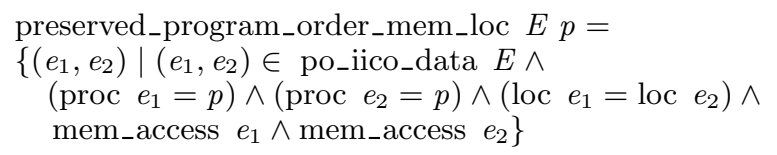

Finally, we collect together the above, together with the intra-instruction data and control relations (restricted to the processor $p$ in question):

$$
\begin{aligned}
& \text { preserved_program_order } E p= \\
& \begin{array}{r}
\left\{\left(e_{1}, e_{2}\right) \mid\left(e_{1}, e_{2}\right) \in \text { E.intra_causality_data } \wedge\right. \\
\left.\quad\left(\text { proc } e_{1}=p\right) \wedge\left(\text { proc } e_{2}=p\right)\right\} \cup \\
\left\{\left(e_{1}, e_{2}\right) \mid\left(e_{1}, e_{2}\right) \in \text { E.intra_causality_control } \wedge\right. \\
\left.\quad\left(\text { proc } e_{1}=p\right) \wedge\left(\text { proc } e_{2}=p\right)\right\} \cup
\end{array}
\end{aligned}
$$

address_or_data_dependency_load_load $E p \cup$

address_or_data_or_control_dependency_load_store $E p \cup$ preserved_program_order_mem_loc $E p$

Note that program order between two register accesses, even to the same register, is not automatically preserved (but only if there is a local_register_data_dependency as above). The documentation [Pow07, p.414] states "Because processors may implement nonarchitected duplicates of architected resources (e.g. GPRs, CR fields, and the Link Register), resource dependencies (e.g. specification of the same target register for two Load instructions) do not order storage accesses." This is illustrated by the ppc.reg example in $\S 4$, which our model admits.

\subsection{Barriers}

To make concurrent programming feasible, given the liberal reordering of normal operations that is permitted on Power and ARM (as described above), both architectures provide a variety of synchronisation instructions. Power 2.05 provides sync L, where L can be 0 ("heavyweight sync"), 1 ("lightweight sync") or 2 (in the Server Environment specification only); an eieio (Server) or mbar MO (Embedded), sharing the same opcode, intended for memory-mapped I/O; and an isync, to synchronise the instruction stream. For the fragment of the ISA we consider, the guarantees provided by sync 0 are the same as those of mbar 0. Broadly, an eieio provides weaker synchronisation, for stores only; an lwsync provides synchronisation for memory accesses except store/load pairs, and mbar for MO $\neq 0$ is implementationdefined. ARMv7 provides DMB ("Data Memory Barrier"), very similar to the Power sync 0 , and the stronger DSB ("Data Synchronisation Barrier") which also synchronises the execution stream, including cache, branch predictor, and TLB maintenance operations. We formalise the Power sync 0 and ARM DMB.

Each instance of such a barrier instruction in an execution defines two groups of memory access events: Group A, in some sense those 'before' the barrier, and Group B, in some sense 'after' the barrier. It ensures that all members of Group A precede all members of Group B, in the view orders of all processors. To express this, we start by defining those instructions to generate a special BARRIER SYNC event, which appears (only) in the view order of the processor that executed the instruction. 


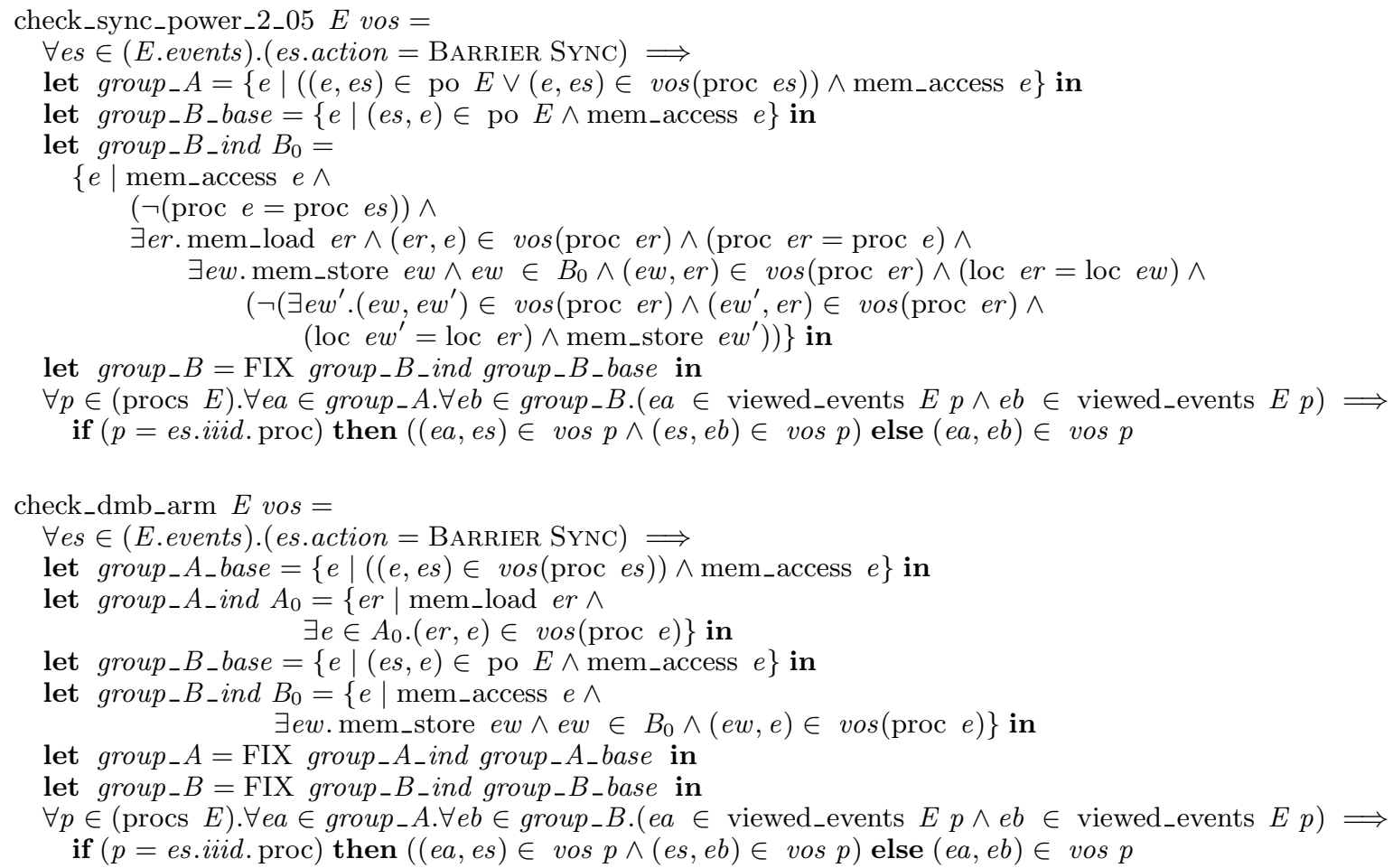

Figure 2. Barrier Semantics

For Power, given such a barrier event es from processor $p$, Group A consists of all memory access events that precede $e s$ in either program order or in $p$ 's view order. Group B is defined inductively. It includes:

- all memory access events that follow $e s$ in the $p$ program order; and

- all memory access events $e$, on other processors $p^{\prime}$, that follow (in the $p^{\prime}$ view order) a memory read event er (by $\left.p^{\prime}\right)$ that reads the value from a memory write event ew in Group B (i.e., $e r$ follows $e w$ in the $p^{\prime}$ view order, they are to the same location, and there is no intervening write $e w^{\prime}$ to the same location in that view order).

For any $e a$ in Group A and $e b$ in Group B, and for all processors, whenever $e a$ and $e b$ are in the viewed_events for that processor (i.e. they are either memory reads by that processor, or memory writes by any processor), we require that $e a$ precedes $e b$ in that processor's view order. For the processor that executed the synchronisation instruction, we also require that the barrier event lies between $e a$ and $e b$. The HOL statement of this is in Figure 2.

For ARM, the Architecture Reference Manual [ARM08a] and the Barrier Litmus Test Cookbook [ARM08b] give two distinct specifications. The Cookbook specification matches the Power semantics we describe above, except that there is no requirement that $p^{\prime} \neq p$. It may be that this (rather strange) requirement is an error in the Power 2.05 specification - the text there speaks of "processors and mechanisms other than P1" [Pow07, II,p.413], but perhaps means "in addition to P1".

The ARM Architecture Reference Manual specification is quite different. Its Group A is also inductive, with a base case of all memory access events that precede $e s$ in $p$ 's view order (but apparently not including those preceding es in program order), and an inductive case adding memory reads er by any processor that precede an event $e$ in Group A in that processor's view order. Its Group B base case is the same as in Power, but its Group B inductive case adds all memory accesses $e$ that follow, in the view order of proc $e$, a memory write in Group B. This is also formalised in Figure 2, and at present our top-level definition uses this, not the Cookbook version, for ARM.

\subsection{A-Cumulativity and 'Performed'}

The Power specification [Pow07, p.413] says the ordering provided by a barrier is "cumulative" if

"it also orders storage accesses that are performed by processors and mechanisms other than P1, as follows.

- A includes all applicable storage accesses by any such processor or mechanism that have been performed with respect to $\mathrm{P} 1$ before the memory barrier is created.

- B includes all applicable storage accesses by any such processor or mechanism that are performed after a load instruction executed by that processor or mechanism has returned the value stored by a store that is in B."

and that sync is indeed cumulative. Here "performed" is defined informally as follows [Pow07, p.408]:

"A load or instruction fetch by a processor or mechanism $(\mathrm{P} 1)$ is performed with respect to any processor or mechanism (P2) when the value to be returned by the load or instruction fetch can no longer be changed by a store by $\mathrm{P} 2$. A store by $\mathrm{P} 1$ is per- 

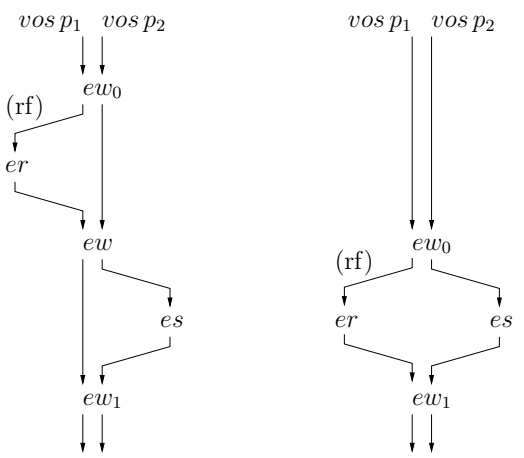

Figure 3. Additional A-cumulativity read cases er

formed with respect to $\mathrm{P} 2$ when a load by P2 from the location accessed by the store will return the value stored (or a value stored subsequently). [...] The preceding definitions apply regardless of whether P1 and P2 are the same entity."

This does not lend itself to a straightforward direct formalisation, for two reasons. First, it implicitly refers to global time. This is not a real problem, as we only need to consider when one access is performed before or after another. Second, more seriously, it is subjunctive: the first clause refers to a hypothetical store by $\mathrm{P} 2$, and the second to a hypothetical load by P2. Our formalisation defines when a specific concrete execution is admitted, and it would be very awkward to express conditions in terms of modified versions of that execution with such hypothetical accesses added.

In most cases where "performed" is used in the informal specification, one is asking whether one memory access $e_{1}$ is performed before another $e_{2}$ with respect to some processor $p$, where both accesses are either writes (by any processor) or reads by $p$. It then seems sufficient to interpret " $e_{1}$ is performed before $e_{2}$ w.r.t $p$ " by $\left(e_{1}, e_{2}\right) \in \operatorname{vos} p$, i.e. that $e_{1}$ precedes $e_{2}$ in $p$ 's view order. ${ }^{2}$

The only exception is for A-cumulativity, where we believe that the Figure 2 definition of group $_{-} A$ is incomplete ${ }^{3}$. Two alternative plausible extensions can be phrased in terms of our view orders.

Conservatively, one could add the reads er by any other processor $p_{1}$ which are before (in the $p_{1}$ view order) some write $e w$, to the same address, that precedes the sync es (in the syncing processor $p_{2}$ 's view order). Such a read er must take its value from some earlier write (or the initial state).

$$
\begin{aligned}
& \{\text { er } \mid \text { mem_load } \text { er } \wedge(\neg(\text { proc er }=\text { proc es })) \wedge \\
& \exists e w \text {. mem_store } e w \wedge(\text { loc } e w=\operatorname{loc} e r) \wedge \\
& (e w, e s) \in \operatorname{vos}(\operatorname{proc} e s) \wedge \\
& (e r, e w) \in \operatorname{vos}(\text { proc } e r)\}
\end{aligned}
$$

More liberally, one could also include reads er that read from the last write $e w_{0}$ (to that location) before the sync, or, in other words, the reads that do not read from a write after the sync:

\footnotetext{
${ }^{2}$ Recall that our view orders do not include memory reads by other processors. This choice seems simpler to us w.r.t. the programmer's intuition, though perhaps it is counterintuitive from an architect's point of view, thinking in terms of cache-line ownership in global time.

${ }^{3}$ Thanks to Paul McKenney for this observation.
}

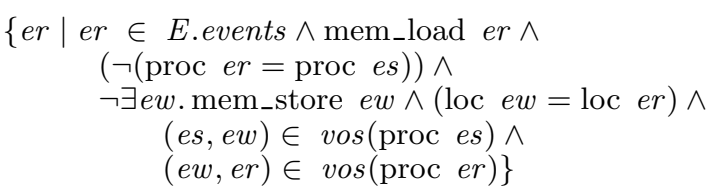

These are illustrated in Figure 3 (with (rf) indicating the reads-from relationships). At present we do not know whether either of them matches the architect's intentions.

Another, more radical, possibility would be to add otherprocessor's reads to the view orders, but one would then also need subtle conditions constraining where in the view orders they appear.

\subsection{Reservations}

The Power and ARM instruction sets both include loadreserve and store-conditional instructions, e.g. 1warx/stwcx and LDREX/STREX. These are intended to be used in pairs: a load-reserve loads a value from a memory address and establishes a reservation for the memory granule including that address; to a first approximation, a later store-conditional by the same processor to the same address succeeds iff no other processor has written to that memory granule since.

We express this semantics with the two special locations per processor: LOCATION_RES (notionally carrying just one bit, but embedded in a word32), and LOCATION_RES_ADDR (carrying the reserved address). The latter behaves just like a register, though in the instruction semantics it is only read when the former is 1 ( $1 w$ in HOL). The former, LOCATION_RES, can be read and written by load-reserve and store-conditional instructions, but the value read is not the most recent value written, instead being computed by location_res_value below. This walks over the relevant view order to find the most recent (in that view order) reservation, if any, and checks that there has been no intervening (in that view order) memory write to the same reservation granule.

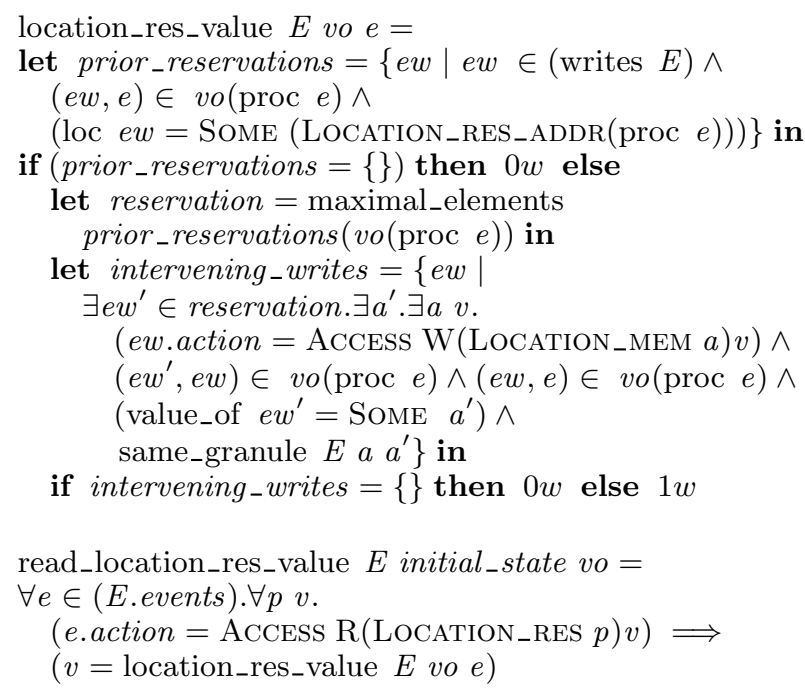

Additionally, we need to ensure that accesses to these special locations, and any associated memory access, are atomic. We record this in the atomicity field of an event structure, and check it with the predicate below. 
check_atomicity $E$ vo $=$

$\forall p \in($ procs $E) . \forall e s \in$ (E.atomicity).

$\forall e_{1} e_{2} \in$ es. $\left(e_{1}, e_{2}\right) \in($ vo $p) \Longrightarrow$

$$
\forall e .\left(e_{1}, e\right) \in(\text { vo } p) \wedge\left(e, e_{2}\right) \in(\text { vo } p) \Longrightarrow e \in e s
$$

Note that any matching load-reserve/store-conditional pair will necessarily be to the same address, and so the definition of preserved_program_order_mem_loc will apply. However, because the value of LOCATION_RES read is computed specially, as above, we do not introduce any dependencies between the events of such a pair and an intervening write by another processor.

\subsection{Valid Executions}

Finally we can define when an execution $X$ over an event structure $E$ is valid. This simply collects together the axioms stated above, together with three conditions preventing intervening register writes between a preserved write/read pair. In brief: the view orders are well formed; read events read the most recent value written; the write serialisation is a proper candidate; for each processor $p$, its preserved coherence order is in the write serialization; for each processor $p$, its view order contains the write serialization, preserved program order, and local register data dependency; there are no intervening writes between a register write and read from local register data dependency, and similarly for register writes before a read from the initial state or write to the final state; the sync or DMB condition is checked; the reservation values are correct; and the atomicity relation is respected.

valid_execution $E X=$

view_orders_well_formed $E X$.vo $\wedge$

read_most_recent_value $E X$.initial_state $X$.vo $\wedge$

$X$.write_serialization $\in$ write_serialization_candidates $E \wedge$

$(\forall p \in($ procs $E)$.

preserved_coherence_order $E p \subseteq X$.write_serialization $\wedge$

$X$.write_serialization $\subseteq X$.vo $p \bar{\wedge}$

preserved_program_order $E p \subseteq X$.vo $p \wedge$

$(*$ no intervening writes in local register data dependency*)

local_register_data_dependency $E p \subseteq X$.vo $p \wedge$

$\left(\forall\left(e_{1}, e_{2}\right) \in\right.$ (local_register_data_dependency $\left.E p\right)$

$\neg\left(\exists e_{3} .\left(e_{1}, e_{3}\right) \in X\right.$.vo $p \wedge\left(e_{3}, e_{2}\right) \in X$.vo $p \wedge$ $\left(\operatorname{loc} e_{3}=\operatorname{loc} e_{1}\right) \wedge$ store $\left.\left.\left.e_{3}\right)\right)\right) \wedge$

$\left(*^{*}\right.$ no intervening writes before a reg read from initial state $\left.{ }^{*}\right)$

$(\forall e \in($ E.events).(reg_load $e \wedge$

$\left(\neg\left(\exists e_{0} \cdot\left(e_{0}, e\right) \in\right.\right.$ po_iico_both $E \wedge$ reg_store $e_{0} \wedge$ $\left.\left.\left.\left(\operatorname{loc} e_{0}=\operatorname{loc} e\right)\right)\right)\right) \Longrightarrow$

$\left(\neg\left(\exists e_{0} \cdot\left(e_{0}, e\right) \in X . v o(\right.\right.$ proc $e) \wedge$ reg_store $e_{0} \wedge$ $\left.\left.\left.\left(\operatorname{loc} e_{0}=\operatorname{loc} e\right)\right)\right)\right) \wedge$

(*no intervening writes after a reg write to the final state*)

$(\forall e \in($ E.events).(reg_store $e \wedge$

$\left(\neg\left(\exists e_{1} .\left(e, e_{1}\right) \in\right.\right.$ po_iico_both $E \wedge$ reg_store $e_{1} \wedge$ $\left.\left.\left.\left(\operatorname{loc} e_{1}=\operatorname{loc} e\right)\right)\right)\right) \Longrightarrow$

$\left(\neg\left(\exists e_{1} .\left(e, e_{1}\right) \in X . v o(\operatorname{proc} e) \wedge\right.\right.$ reg_store $e_{1} \wedge$ $\left.\left.\left.\left(\operatorname{loc} e_{1}=\operatorname{loc} e\right)\right)\right)\right) \wedge$

(case E.arch of

POWER205 $\rightarrow$ check_sync_power_2_05 E X.vo

$\|$ ARMv7 $\rightarrow$ check_dmb_arm $E X$.vo $) \wedge$

read_location_res_value $E X$.initial_state $X$.vo $\wedge$

check_atomicity $E X$.vo

\section{$2.9 \quad$ Example}

In Fig. 4 we show a simple example of a valid execution, produced by memevents, for the ppc3.1 program below. Here we store to two different locations, on two processors, and

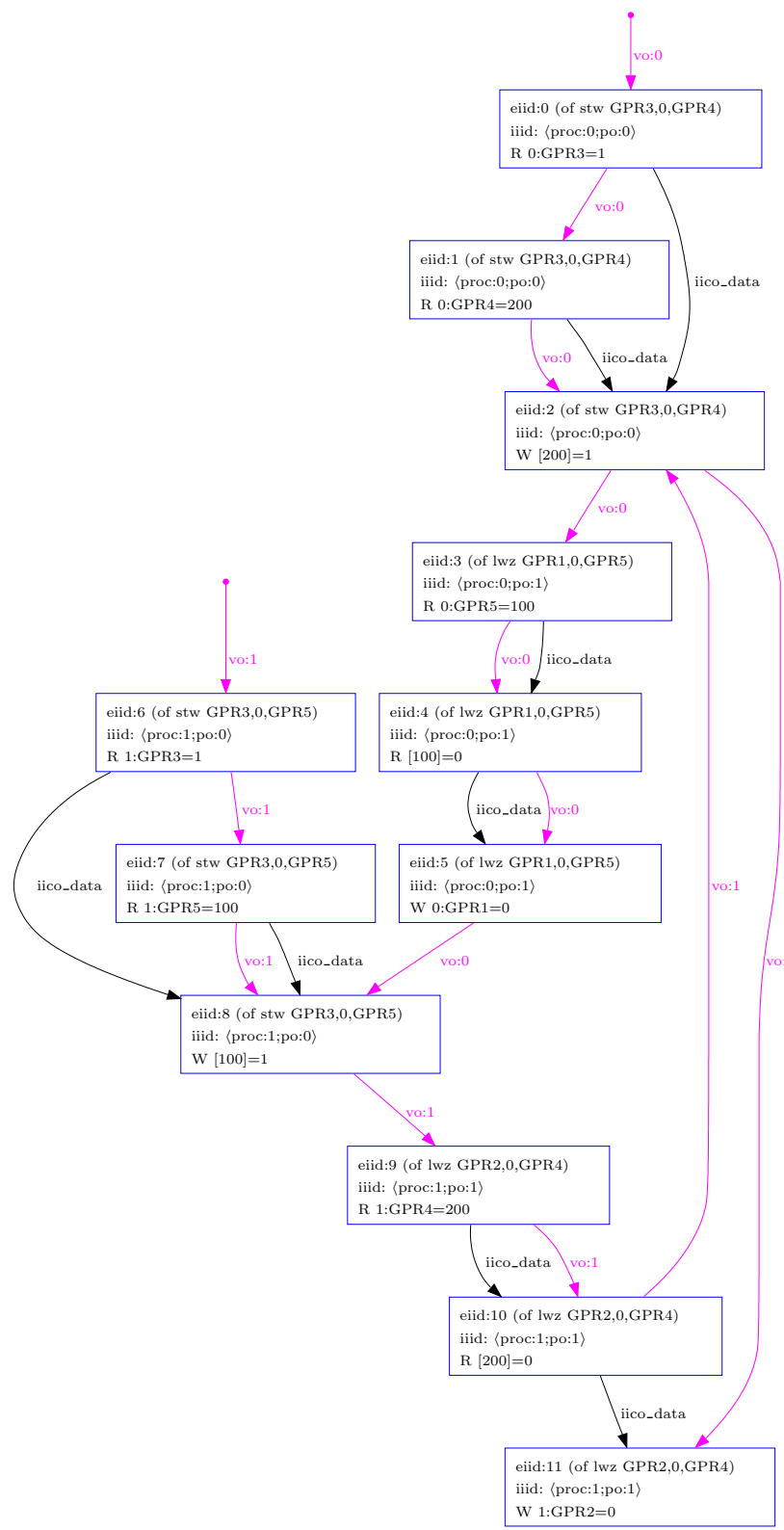

ppc3.1: (event structure 1)

Figure 4. A Valid Execution (ppc3.1)

each reads from the other location. There are very few dependencies in this example, and, in the valid execution shown, both reads are from the initial state. Note that the union of the two view orders (vo:0 and vo:1) is cyclic.

\begin{tabular}{|l|c|l|}
\hline ppc3.1 & \multicolumn{1}{|c|}{ proc:0 } & \multicolumn{1}{c|}{ proc: 1} \\
\hline poi:0 & stw GPR3,0,GPR4 & stw GPR3,0,GPR5 \\
poi:1 & lwz GPR1,0,GPR5 & lwz GPR2,0,GPR4 \\
\hline Initial state: $0: G P R 3=1 ; \quad 0: G P R 4=200 ; \quad 0: G P R 5=100 ;$ \\
1:GPR3=1; $1: G P R 4=200 ; 1: G P R 5=100$ (elsewhere 0) \\
\hline \multicolumn{2}{|l|}{ Allowed: $0: G P R 1=0 \wedge 1: G P R 2=0$} \\
\hline
\end{tabular}




\section{Instruction Semantics}

As in the x 86 model, the overall semantics is factored into two parts: the instruction semantics defines, for any program, a set of candidate event structures, and the axiomatic memory model of the previous section defines, for each event structure, its valid executions.

\subsection{ARM}

At the time of writing there are seven versions of the ARM instruction set architecture, ARMv1 through ARMv7. ${ }^{4}$ The ARM11 MPCore and ARM Cortex-A9 MPCore are ARMv6 and ARMv7 architectures respectively. Versions one (never used in a commercial product) and two are now obselete. Each successive version has provided extensions and minor revisions to the previous version. The revisions have been mostly conservative; for example, behaviours previously categorised as unpredictable, or implementation dependent, have been specified later as undefined (which is handled by an well defined exception entry mechanism) or provided with a semantics.

Architecture ARMv3 was specified by Fox in HOL when verifying a model of the ARM6 micro-architecture [Fox03]. This ISA model was later extended to ARMv4 and that model has been used by Myreen et al. to verify machine code programs [MSG08]. Architecture ARMv4T was subsequently specified, in its entirety, in HOL. These HOL models are conventional, deterministic, functional specifications and are not immediately suited to reasoning about weak memory models. Thus, we have converted and extended these ISA models into a single monadic style specification (covering ARMv3 to ARMv5TE, together with LDREX/STREX from ARMv6 and DMB from ARMv7). The monadic specification supports non-determinism and reasoning about register and memory access events. ARMv6 introduced around 100 other new instructions, but these were mostly extra arithmetic and bit-manipulations (also extra exclusive loads/stores, for bytes, half-words, etc.); our semantics therefore covers essentially all the interesting concurrency cases.

\subsection{Power}

Our instruction semantics for Power is a HOL4 version of Leroy's specification of PowerPC assembly [Ler06], extended with the Iwarx, stwcx, and sync instructions. Leroy's model was translated into HOL4 and a instruction decoder was attached to it in order to make his assembly-level model a machine-level model. The specification defines instructions: add, addi, addis, addze, and., andc, andi., andis., b, bctr, bctrl, bf, bl, bs, blr, bt, cmplw, cmplwi, cmpw, cmpwi, cror, eqv, extsb, extsh, lbz, lbzx, lha, lhax, lhz, lhzx, lwarx, lwz, lwzx, mflr, mr, mtctr, mtlr, mulli, mullw, nand, nor, or, orc, ori, oris, slw, sraw, srawi, srw, stb, stbx, sth, sthx, stw, stwcx, stwx, subfc, subfic, sync, xor, xori, xoris. Floating-point instructions were omitted.

We have a reasonable level of confidence in the correctness of these instruction semantics: for ARM, based on the verification of the ARMv3 fragment against a microarchitectural model, and Myreen et al.'s verification of machine-code programs above the semantics; for Power, based on Leroy et al.'s extensive work. For both, further confidence will come from empirical testing: as we did for $\mathrm{x} 86\left[\mathrm{SSZN}^{+} 09\right]$, we intend to automatically generate $\mathrm{HOL}$ conjectures from in-

\footnotetext{
${ }^{4}$ There are also numerous optional extensions (variants) to these architectures. For example: Thumb, Thumb-2, DSP and Jazelle extensions.
}

strumented execution of instructions on actual processors, and to (automatically) prove that these conjectures are true in the semantics.

\section{Litmus tests}

Memory models are often illustrated (or even 'defined') in terms of behaviours of small litmus-test programs that are either allowed, required, or forbidden. In this section we present a few such tests, as space permits; many more will be required to give reasonable coverage of our model.

Total order on writes to the same location In litmus test ppc6, in Fig. 5 (an analogue of the x86 $\left[\mathrm{SSZN}^{+} 09\right.$, iwp2.6]), two processors concurrently update the same memory location (100, in GPR2) with two different values (1 and 2 ), while two other processors read twice from that memory location. Coherence $(\S 2.3)$ requires the existence of a strict linear order on all the stores to one location, and this order must be preserved by all the view orders. This implies that the view orders of processors 2 and 3 must agree on the order in which they see the stores performed by processors 0 and 1 , forbidding the final state reported in the figure.

Local reordering of stores or loads to different locations The ARM and Power architectures allow processors to reorder their individual memory accesses rather freely. Test ppc1 below shows that a pair of stores, or a pair of loads, to non-overlapping different memory locations, can be reordered. The specified outcome can be obtained by a possible execution in which the two loads performed by proc: 1 are executed in the opposite order to program order; or alternatively a possible execution in which the two stores performed by proc:0 are reordered. This is an analogue of $\left[\mathrm{SSZN}^{+}\right.$09, iwp2.1/amd1], except that this "Allowed" outcome is forbidden there.

\begin{tabular}{|l|c|l|}
\hline ppc1 & proc:0 & \multicolumn{1}{c|}{ proc:1 } \\
\hline poi:0 & stw GPR1,0,GPR5 & lwz GPR3,0,GPR6 \\
poi:1 & stw GPR2,0,GPR6 & lwz GPR4,0,GPR5 \\
\hline Initial & state: 0:GPR1 $=1 ; \quad 0: G P R 2=1 ; \quad 0: G P R 5=200 ;$ \\
0:GPR6=100; 1:GPR5=200; 1:GPR6 $=100$ (elsewhere 0) \\
\hline Allowed: 1:GPR3=1 $\wedge$ 1:GPR4=0 \\
\hline
\end{tabular}

Local reordering of stores and loads to different locations Test ppc3.1 (analogue of $\left[\mathrm{SSZN}^{+}\right.$09, iwp2.3.a/amd4]) in $\S 2.9$ shows that non-overlapping memory stores and loads can be reordered.

Write buffering In test ppc4 (an analogue of $\left[\mathrm{SSZN}^{+} 09\right.$, iwp2.4/amd9]) each processor might see the other memory store at the end of their view order, thus admitting the outcome shown.

\begin{tabular}{|l|c|l|}
\hline ppc4 & proc:0 & \multicolumn{1}{c|}{ proc:1 } \\
\hline poi:0 & stw GPR1,0,GPR4 & stw GPR1,0,GPR5 \\
poi:1 & lwz GPR2,0,GPR4 & lwz GPR2,0,GPR5 \\
poi:2 & lwz GPR3,0,GPR5 & lwz GPR3,0,GPR4 \\
\hline Initial state: $0: G P R 1=1 ; \quad 0: G P R 4=200 ; \quad 0: G P R 5=100 ;$ \\
1:GPR1=1; $1: G P R 4=200 ; \quad 1: G P R 5=100$ (elsewhere 0) \\
\hline \multicolumn{2}{|l|}{ Allowed: 0:GPR3 $=0 \wedge 1: G P R 3=0$} \\
\hline
\end{tabular}

Shadow registers Test ppc.reg (Adir et al. [AAS03, Test 6]) illustrates that the existence of shadow registers is observable. The specified outcome can be obtained only by an execution in which proc:0 executes the $1 i$ and stw instructions before the $1 \mathrm{wz}$ and $\mathrm{mr}$ ones. Observe however 
Recent work on memory models for high-level languages, including Java, X10, and C++ [MPA05, AS07, SJMvP07, BA08], is also relevant. There one must deal not just with reordering by the processor, but also arising from compiler optimizations. We hope that having precise descriptions of the underlying architecture will assist such work.

\section{Discussion}

We have presented a semantics for multiprocessor programs above the Power and ARM architectures.

In this final section we introduce several criteria that such a semantics should satisfy, and discuss the extent to which our semantics does so.

First, it should be precise. It may be a loose specification, but it should unambiguously define what is and is not permitted. Ours is expressed in mechanised mathematics, in a well-defined logic, and using a proof assistant (HOL).

Second, it should have good coverage. We do not attempt to model the entirety of each architecture (to do so, including all the page-table operations etc., would be a mammoth task). However, we do model a large enough fragment for much low-level concurrent programming, including the memory model for "Normal" (ARM) and "Memory Coherence Required" (Power) memory.

Third, it should be accurate with respect to the architecture. This is a subtle point, as the architectures are informal prose, not themselves precisely defined objects - indeed, establishing such precise definitions is the main goal of this paper. Nonetheless, the semantics should be informally sound with respect to the architecture, i.e. any behaviour that is admitted by the informal specification should be admitted by the formal specification. The semantics might be looser than the informal specification, but not too much so, otherwise it may fail to capture a property that programmers need. We have taken care to interpret the informal prose specifications as best we can. We have described our model here in its own terms, for clarity and brevity, but in a longer version of this paper we intend to spell out the correspondence between the informal prose and our specification. For example, the Power and ARM specifications speak in terms of actions being "performed" or "observed"; these concepts generally are modelled by our view orders.

Fourth, it should be accurate with respect to processor implementations. This can be tested empirically, as we did for $x 86$, and we intend to do so again here. In principle, it can also be established by proof, showing that the semantics is an accurate abstraction of a microarchitectural model (e.g. as in Fox's verification for ARM6 w.r.t ARMv3 [Fox03]). That would be highly desirable, but very challenging: for modern multiprocessors, such a model would be very large, and also proprietary.

Fifth, it should be strong enough for reasonable programs. The semantics should constrain the behaviour of the processors enough that reasonable programs can be shown to have their intended behaviour. This applies also to the informal specifications, and there it is almost impossible to assess: one simply cannot determine all the possible behaviours of a non-trivial program, by hand, against those documents. For our precise model it should be possible to prove (for small programs) how they behave, and to test larger programs against a nondeterministic emulator which can exhibit all valid executions. It should also be possible to prove general metatheoretic results, e.g. that programs that are (in some model-specific sense) data-race-free behave sequentially consistently.
Sixth, it should be accessible, to low-level programmers, builders of verification tools, language designers and implementors, and hardware architects, as the interface between these four groups. Our axiomatic model is moderately complex, but we believe this reflects the complexity of the intended vendor architecture. It is written in relatively straightforward typed logic, and can be stated in full in only a few pages. Time will tell whether this, augmented by further examples, suffices. Certainly having a precise mathematical specification should make it easier to write selfconsistent tutorial documents. We have primarily targeted the first three groups, writing a specification that is relatively free of microarchitectural concepts.

Acknowledgements We thank Nathan Chong for discussions about the ARM, and Luc Maranget for comments on a draft, Paul McKenney and Raul Silvera for comments on the PowerPC, and Doug Lea. We acknowledge the support of EPSRC grants GR/T11715, EP/C510712, and EP/F036345, and ANR grant ANR-06-SETI-010-02.

\section{References}

[AAS03] A. Adir, H. Attiya, and G. Shurek. Information-flow models for shared memory with an application to the powerpc architecture. IEEE Trans. Parallel Distrib. Syst., 14(5):502-515, 2003.

[AG96] S.V. Adve and K. Gharachorloo. Shared memory consistency models: A tutorial. IEEE Computer, 29(12):66-76, Dec 1996.

[ARM08a] ARM. ARM Architecture Reference Manual (ARMv7$A$ and $A R M v 7-R$ edition). 2008. Available from ARM.

[ARM08b] ARM. ARM Barrier Litmus Tests and Cookbook, February 2008.

[AS07] D. Aspinall and J. Sevcik. Formalising Java's data race free guarantee. In Proc. TPHOLs, LNCS, 2007.

[BA08] H.-J. Boehm and S.V. Adve. Foundations of the C++ concurrency memory model. SIGPLAN Not., 43(6):6878, 2008.

[CI08] Nathan Chong and Samin Ishtiaq. Reasoning about the ARM weakly consistent memory model. In Proc. MSPC, 2008.

[Coq] The Coq proof assistant. http://coq.inria.fr/.

[CSB] F. Corella, J. M. Stone, and C. M. Barton. A formal specification of the PowerPC shared memory architecture. Technical Report RC18638, IBM.

[Fox03] Anthony Fox. Formal specification and verification of ARM6. In Proc. TPHOLs, Theorem Proving in Higher Order Logics, LNCS 2758, pages 25-40, 2003.

[HJK06] L. Higham, L. A. Jackson, and J. Kawash. Programmercentric conditions for itanium memory consistency. In Proc. ICDCN, 2006.

[HOL] The HOL 4 system. http://hol. sourceforge.net/.

[IBM02] IBM. Book E - Enhanced PowerPC Architecture. May 2002.

[Ita] A formal specification of Intel Itanium processor family memory ordering. http://developer.intel. com/design/itanium/downloads/251429.htm.

[Ler06] Xavier Leroy. Formal certification of a compiler backend, or: programming a compiler with a proof assistant. In Proc. POPL, 2006.

[LHF05] Michael Lyons, Bill Hay, and Brad Frey. PowerPC Storage Model and AIX Programming. November 2005.

[LJV97] L.Higham, J.Kawash, and Nathaly Verwaal. Defining 
and comparing memory consistency models. In PDCS, 1997.

[Luc01] V. M. Luchangco. Memory consistency models for highperformance distributed computing. PhD thesis, MIT, 2001.

[MPA05] J. Manson, W. Pugh, and S.V. Adve. The Java memory model. In Proc. POPL, 2005.

[MSG08] Magnus O. Myreen, Konrad Slind, and Michael J. C. Gordon. Machine-code verification for multiple architectures: An application of decompilation into logic. In Proc. FMCAD, Formal Methods in Computer-Aided Design, 2008. To appear.

[PD95] S. Park and D. L. Dill. An executable specification, analyzer and verifier for RMO (relaxed memory order). In Proc. SPAA' '95, 1995.

[Pow07] Power ISA Version 2.05. October 2007.

[SF95] Janice M. Stone and Robert P. Fitzgerald. Storage in the PowerPC. IEEE Micro, 1995.

[SJMvP07] V.A. Saraswat, R. Jagadeesan, M. Michael, and C. von Praun. A theory of memory models. In Proc. PPoPP, 2007.

[Spa] The SPARC architecture manual, v. 9. http:// developers.sun.com/solaris/articles/sparcv9.pdf.

$\left[\mathrm{SSZN}^{+}\right.$09] S. Sarkar, P. Sewell, F. Zappa Nardelli, S. Owens, T. Ridge, T. Braibant, M. Myreen, and J. Alglave. The semantics of x86-cc multiprocessor machine code. In Proc. POPL 2009, January 2009. To appear.

[wea08] The semantics of multiprocessor machine code, 2008. www.cl.cam.ac.uk/users/pes20/weakmemory.

[YGLS04] Y. Yang, G. Gopalakrishnan, G. Lindstrom, and K. Slind. Nemos: A framework for axiomatic and executable specifications of memory consistency models. In IPDPS, 2004. 